\title{
Gradhiva
}

\section{Luc Boltanski et Arnaud Esquerre, Enrichissement : une critique de la marchandise}

Paris, Gallimard, coll. « NRF Essais », 2017

\section{Frédéric Keck}

\section{OpenEdition}

\section{Journals}

Édition électronique

URL : https://journals.openedition.org/gradhiva/3354

DOI : $10.4000 /$ gradhiva.3354

ISSN : 1760-849X

\section{Éditeur}

Musée du quai Branly Jacques Chirac

Édition imprimée

Date de publication : 31 mai 2017

Pagination : 266-269

ISBN : 978-2-35744-095-1

ISSN : 0764-8928

\section{Référence électronique}

Frédéric Keck, «Luc Boltanski et Arnaud Esquerre, Enrichissement : une critique de la marchandise »,

Gradhiva [En ligne], 25 | 2017, mis en ligne le 31 mai 2017, consulté le 21 septembre 2021. URL : http:// journals.openedition.org/gradhiva/3354 ; DOI : https://doi.org/10.4000/gradhiva.3354

Ce document a été généré automatiquement le 21 septembre 2021.

(c) musée du quai Branly 


\title{
Luc Boltanski et Arnaud Esquerre, Enrichissement : une critique de la marchandise
}

\author{
Paris, Gallimard, coll. « NRF Essais », 2017
}

Frédéric Keck

\section{RÉFÉRENCE}

Luc Boltanski et Arnaud Esquerre, Enrichissement : une critique de la marchandise. Paris, Gallimard, coll. « NRF Essais », 2017, 672 p. 


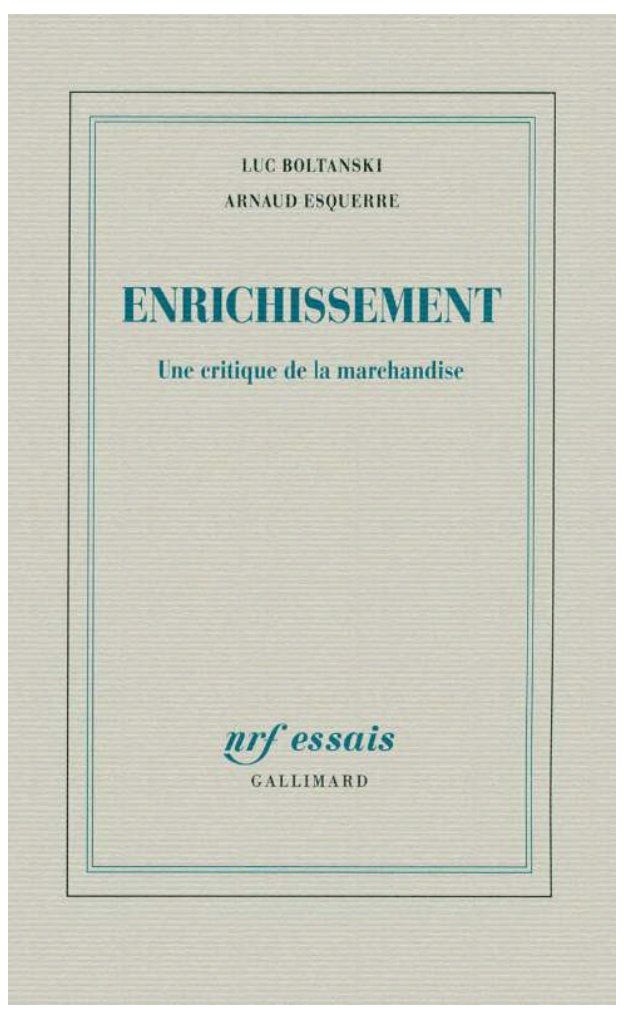

Le livre de Luc Boltanski et d'Arnaud Esquerre est une intervention dans le champ de l'anthropologie de l'art depuis la sociologie et l'économie. Frère du célèbre artiste Christian Boltanski, Luc Boltanski étudie depuis trente ans les transformations sociales de la modernité, notamment avec ses analyses sur l'émergence de la catégorie socioprofessionnelle de cadre et sur la formation d'un "nouvel esprit du capitalisme ${ }^{1}$ ». À partir des manuels de management adressés aux classes dirigeantes, il montre comment des modalités d'interaction sont justifiées par des valeurs, notamment à travers la tendance croissante à l'évaluation. Dans son dernier ouvrage, il se penche sur la notion de marchandise qui, depuis les analyses de Karl Marx au XIX ${ }^{e}$ siècle, est au cœur des transformations du capitalisme.

2 La thèse des deux auteurs peut être résumée ainsi : la marchandise n'est pas une forme d'organisation spécifique au mode de production industriel, c'est une modalité d'interaction universelle entre les hommes et les choses. En cela, cette thèse est anthropologique, même si elle est appliquée à un diagnostic de la société française contemporaine. Les deux sociologues, en s'appuyant sur les travaux récents des économistes, critiquent les anthropologues sur deux points : soit ceux-ci opposent à l'économie de marché une sphère d'échanges symboliques qui lui échappe, soit ils projettent sur d'autres sociétés des modèles économiques issus du capitalisme occidental $^{2}$. En se réclamant du structuralisme de Claude Lévi-Strauss ${ }^{3}$, Boltanski et Esquerre étudient les transformations des arrangements et des bricolages par lesquels les individus donnent sens aux choses qu'ils ont entre les mains. Ils proposent ainsi la définition la plus large de la notion de marchandise : « toute chose à laquelle échoit un prix quand elle change de propriétaire. » (p. 12)

L'innovation conceptuelle majeure proposée par cet ouvrage est en effet la distinction entre le prix et la valeur. À la suite des analyses de Karl Marx, les économistes ont en effet beaucoup discuté la distinction entre la valeur d'usage et la valeur d'échange 
d'une chose, celle-ci étant justifiée par le marché tandis que celle-là serait justifiée par le travail - ce qui conduit Marx à dénoncer une plus-value produite par le travail et aliénée/exploitée par le marché. Cette thèse conduit Marx, selon Boltanski et Esquerre, à substantiver la valeur en la mettant dans la marchandise elle-même alors que, selon eux, elle résulte toujours d'une transaction à l'issue incertaine entre des personnes. Le prix est ce qui justifie la valeur d'une marchandise : c'est un point d'arrêt dans le processus d'évaluation auquel se livrent des personnes engagées dans une transaction: «Une chose, n'importe laquelle, se transforme en marchandise quand, dans une situation d'échange, un prix lui échoit. » (p. 110)

4 Le prix est donc à la fois réel, mesuré et circonstancié : il vaut pour telle modalité de transaction à tel moment mais peut varier si les rapports de force qui s'instaurent entre les partenaires de l'échange se transforment. C'est donc un signe au sens que cette notion prend dans l'anthropologie structurale: il n'a pas de contenu en soi mais seulement en relation avec d'autres prix dans un marché (ou toute autre institution garantissant la stabilité des prix). C'est pourquoi Boltanski et Esquerre introduisent la notion de métaprix pour désigner le prix sur lequel un grand nombre d'acteurs s'entendent indépendamment des aléas des échanges. Par exemple, en France, le prix du livre est fixé par un métaprix garanti par l'État, ou les prix des œuvres d'art sont fixés par leur valeur d'assurance lorsqu'elles sont échangées entre des galeries ou des musées (alors même que ces œuvres sont supposées hors du commerce).

5 Les critiques du capitalisme commencent toujours par une contestation des prix, lorsque la valeur d'une chose est supposée supérieure ou inférieure à son prix. Il y a crise du capitalisme lorsque le prix des choses apparaît comme arbitraire, ce qui conduit à opposer des valeurs morales à la réalité des choses. Contre cette opposition classique en sciences sociales, Boltanski et Esquerre montrent que le capitalisme parvient à absorber les critiques qui lui sont faites en variant les formats à travers lesquels les choses acquièrent un prix et une valeur. C'est la seconde thèse essentielle du livre : les valeurs ne s'opposent pas aux choses car il y a une pluralité de formes de mise en valeur; le capitalisme moderne, en inventant la forme marchandise, loin de séparer les choses des valeurs, a plutôt multiplié les formes de mise en valeur pour augmenter le désir d'acquérir des choses et ainsi les potentialités d'enrichissement.

6 Les deux sociologues distinguent quatre formes de mise en valeur des choses qui forment entre elles un groupe de transformation - de façon analogue à la méthode de Philippe Descola qui distingue quatre modes d'identification entre humains et nonhumains ${ }^{4}$. Il s'agit pour eux d'établir des structures élémentaires de la marchandise à la façon dont Claude Lévi-Strauss a analysé des structures élémentaires de la parenté, à partir de cet universel qu'est la possession d'une chose par une personne. Mais il ne s'agit nullement de modules cognitifs ou juridiques stables à travers lesquels la propriété est garantie, car chacune de ces formes de mise en valeur suppose un manque à combler, en sorte qu'elle est inévitablement confrontée au risque de sa mise en faillite.

7 La première est la forme standard promue par le capitalisme industriel et la société de consommation. Elle vise à produire des biens uniformes pour répondre aux besoins des consommateurs. Elle repose sur la distinction entre des prototypes, sur lesquels un droit d'auteur est juridiquement garanti, et des spécimens qui en sont la reproduction. Alors que le prototype est fortement personnalisé, puisqu'il porte souvent le nom de son auteur, les spécimens sont des « objets en soi - simplement des choses et rien 
d'autre - puisque leur part d'humanité est tout entière concentrée dans le prototype " (p. 212). C'est pourquoi la forme standard est critiquée au nom de l'authenticité des personnes comme une forme d'aliénation dans la forme marchandise.

8 La forme collection apparaît alors comme une façon de sauver le capitalisme, puisqu'elle émerge en même temps que cette critique. Si on peut faire remonter la passion des collectionneurs aux cabinets de curiosité de la Renaissance, voire aux désirs et aux obsessions de l'enfance, c'est véritablement au xix siècle - comme en attestent les romans d'Honoré de Balzac et d'Anatole France (p. 244-247) - qu'elle apparaît comme un correctif et un prolongement du capitalisme. C'est parce que le capitalisme produit un nombre toujours croissant de marchandises, et parce que les révolutions technologiques les transforment rapidement en déchets ${ }^{5}$, que des choses dévalorisées dans la forme standard se trouvent revalorisées dans la forme collection. La collection est l'organisation systématique des choses en séries de façon à faire apparaître des manques. "Le souci de posséder certaines pièces pour combler certains manques, définis par référence à une totalité idéelle, constitue l'un des principaux motifs auxquels obéissent les conduites au sein de communautés de collectionneurs. » (p. 69)

$\mathrm{Du}$ fait qu'elle ne peut sauver tous les objets de la forme standard en les considérant comme des objets de collection, du fait qu'elle est nécessairement sélective, voire arbitraire, dans le choix des objets qu'elle sauve, la forme collection doit donc laisser place à une autre forme qui procède comme elle mais en se détachant de la matérialité des objets : ce que Boltanski et Esquerre appellent la forme actif ${ }^{6}$. Celle-ci met en valeur les objets en pariant sur leur potentialité marchande, c'est-à-dire sur leur capacité à engendrer de la monnaie au fur et à mesure qu'ils circulent. La forme actif repose donc sur l'écart entre le prix d'une chose et son métaprix, et s'appuie sur des institutions comme les musées, les galeries d'art ou les maisons de vente aux enchères, qui assurent la stabilité du métaprix. Elle vise donc à maintenir simultanément et de façon contradictoire la liquidité des choses (leur capacité à être transportées et transformées rapidement en monnaie) et leur durabilité (leur conservation au cours du temps). Si n'importe quoi peut être considéré comme un actif, comme on le voit sur le marché de l'art contemporain dont les cotes ne cessent de monter, l'arbitraire de la sélection sur laquelle repose cette valorisation apparaît encore plus nettement que dans la forme collection, puisque le caractère inégalitaire et le coût de conservation de l'art contemporain apparaissent particulièrement criants.

Une quatrième forme peut alors être distinguée, qui retient de la forme collection son caractère narratif tout en se détachant également de la matérialité : la forme tendance. Dans cette forme, la marchandise acquiert une valeur en tant qu'elle suscite un désir dans un marché structuré par les hiérarchies sociales : entre vieux et jeunes, pauvres et riches, ruraux et urbains, etc. Le manque comblé par cette forme est donc celui d'une ascension sociale dans un marché apparemment égalitaire. La critique de la société de consommation dans les années 1960 a particulièrement porté sur cette forme, en tant qu'elle multiplie des signaux d'appartenances sociales faciles à déconstruire. Pourtant la montée d'une industrie du luxe qui a profondément réorganisé l'activité industrielle (comme en atteste l'histoire du groupe LVMH dirigé par Bernard Arnault : p. 304-314) conduit à faire de cette forme un moteur essentiel des transformations actuelles du capitalisme.

11 Chacune de ces quatre formes fonctionne en alternance avec les autres pour requalifier les choses lorsque, sous l'effet de la critique du capitalisme, elles cessent de susciter un 
désir d'acquisition chez les personnes. La thèse de Boltanski et d'Esquerre est en effet la suivante : l'enrichissement, qui est le moteur principal du capitalisme, n'est pas une pathologie ou un mal en soi, mais une façon d'encadrer les relations entre les personnes lorsqu'elles entrent en concurrence pour acquérir des choses. Si toutes les sociétés produisent de l'enrichissement, le capitalisme a multiplié les formats d'enrichissement lorsque, comme l'a montré Fernand Braudel, il a élargi la sphère marchande à l'ensemble de la planète, produisant ainsi de la plus-value marchande entre deux marchés éloignés l'un de l'autre (p. 384). Avec la mondialisation, le marchand est celui qui est capable de connecter deux partenaires très éloignés de l'échange (par exemple un roi africain et un roi allemand lors de la constitution des grandes collections d'art africain à la fin du XIX ${ }^{e}$ siècle) en prenant le point de vue de chacun de ces partenaires (l'ethnologue transformant alors en science désintéressée et universelle ce qui est d'abord une modalité d'échange local visant le profit). Radicalisant cette thèse de Braudel, Boltanski et Esquerre affirment que le capitalisme transforme chaque individu en marchand visant à s'enrichir par la multiplication des formes de la marchandise. Ainsi la collection de timbres, qui était d'abord une activité de jeunes filles au début $d u \mathrm{XIX}^{\mathrm{e}}$ siècle, fut encouragée auprès des garçons car elle leur apprenait à mettre en ordre les choses en leur attribuant une valeur et un prix : « Si l'économie de l'enrichissement s'adresse d'abord aux riches et aux très riches, une de ses spécificités est de s'adresser aux autres comme s'ils étaient riches, ou, à tout le moins, plus riches qu'ils ne le sont. » (p. 65)

Les deux sociologues concluent leur ouvrage par une description des travailleurs précaires du monde de la culture, conçus comme une main-d'œuvre disponible pour l'enrichissement des marchandises puisque, du fait du fort capital culturel produit par l'investissement de l'État dans la politique de la culture, ils peuvent construire des histoires légitimes sur les choses. Si cette analyse conclut le livre sur la mise en lumière d'une exploitation et donc la possibilité d'une émancipation, elle ouvre également, pour l'anthropologie des arts, un nouveau champ de recherche. La notion de patrimoine a en effet été mise au centre des recherches en anthropologie au cours des vingt dernières années pour comprendre comment des objets abandonnés pouvaient être revalorisés par leur "patrimonialisation» (p. 32 sq.). En mettant en avant le travail d'un grand nombre d'acteurs du monde de la culture pour produire des récits transformant les objets en patrimoine, mais aussi les formes d'exploitation qu'elles impliquent pour ces travailleurs et les alternatives qui existent dans les relations entre les personnes et les choses, ce livre ouvre les yeux sur la fabrique des valeurs qui constitue notre monde. Alors que de nombreux analystes mettent en avant un capitalisme dématérialisé dont ils célèbrent les vertus de façon enchantée, Boltanski et Esquerre rappellent que nous n'avons jamais autant été entourés de choses. Le problème aujourd'hui est donc moins de se débarrasser de ces matérialités que de trouver les bons formats pour les mettre en valeur. 


\section{NOTES}

1. Voir Luc Boltanski, Les Cadres: la formation d'un groupe social. Paris, Les Éditions de Minuit, 1982 ; et avec Ève Chiapello, Le Nouvel Esprit du capitalisme. Paris, Gallimard, 1999. Les travaux de Luc Boltanski ont aussi porté sur l'humanitaire (La souffrance à distance : morale humanitaire, médias et politique. Paris, Métailié, 1993) et sur l'avortement (La Condition fotale: une sociologie de l'engendrement et de l'avortement. Paris, Gallimard, 2004) qui peuvent être lus comme des essais sur les transformations morales du capitalisme dans son rapport au vivant. Il est aussi l'auteur d'ouvrages plus réflexifs sur la sociologie : L'Amour et la justice comme compétences: trois essais de sociologie de l'action. Paris, Métaillé, 1990 ; avec Laurent Thévenot, De la justification : les économies de la grandeur. Paris, Gallimard, 1991 ; Rendre la réalité inacceptable : à propos de « La Production de l'idéologie dominante ». Paris, Demopolis, 2008 ; De la critique : précis de sociologie de l'émancipation. Paris, Gallimard, 2009 ; Énigmes et complots : une enquête à propos d'enquêtes. Paris, Gallimard, 2012.

2. La cible principale de l'analyse est le travail de Pierre Bourdieu sur le marché de l'art, accusé de reproduire des distinctions symboliques sans véritablement analyser les mécanismes de formation des prix. Bourdieu croise ainsi les deux travers repérés par Boltanski et Esquerre en analysant des stratégies distinctives dans l'économie des biens symboliques profitant de l'écart temporel entre le don et le contre-don (p. 112-113).

3. Boltanski et Esquerre se réclament d'un structuralisme pragmatique qui, partant de l'expérience ordinaire des personnes, décrit comment elle se divise en valeurs potentiellement conflictuelles dans leur confrontation avec les choses, ces conflits étant réglés par des groupes de transformation sémiotique. Arnaud Esquerre a appliqué cette méthode à plusieurs objets: la religion (La Manipulation mentale: sociologie des sectes en France. Paris, Fayard, 2009), les restes humains (Les Os, les cendres et l'État. Paris, Fayard, 2011) et l'anticipation (Prédire: l'astrologie en France au XXI ${ }^{e}$ siècle. Paris, Fayard, 2013; Théorie des événements extraterrestres: essai sur le récit fantastique. Paris, Fayard, 2016).

4. Voir Philippe Descola, Par-delà nature et culture. Paris, Gallimard, 2005.

5. Si cette analyse semble reprendre la célèbre thèse de Joseph Schumpeter sur la « destruction créatrice " dans le capitalisme, elle prolonge surtout la moins célèbre "théorie du déchet " formulée par Michael Thompson dans Rubbish Theory: the Creation and Destruction of Value. Oxford, Oxford University Press, 1979.

6. «Le dispositif de la collection permet, au même titre que les opérations financières quand elles s'éloignent de l'investissement stricto sensu, d'engendrer de la rareté à partir de tout ou, ce qui revient au même, de rien - y compris de déchets - puisqu'en prenant appui sur la construction de formes sérielles de totalisation, il crée des manques qui réclament impérativement d'être comblés. » (p. 292) Je modifie l'ordre de présentation des quatre formes de mise en valeur dans le livre car il me semble que la forme actif est plus facile à saisir depuis la forme collection que depuis la forme tendance, le passage de l'une à l'autre étant plus évident notamment sur le marché de l'art. 


\section{AUTEURS}

\section{FRÉDÉRIC KECK}

Frederic.KECK@quaibranly.fr 